\title{
Klinischer Stellenwert und Indikationen zur Magnet- resonanztomografie des Herzens 2013: Ein interdisziplinäres Expertenstatement
}

\author{
Clinical Relevance and Indications for Cardiac Magnetic Resonance Imaging \\ 2013: An Interdisciplinary Expert Statement
}

Autoren

Institute
K. Hergan' ${ }^{1}$, S. Globits ${ }^{2}$, H. Schuchlenz ${ }^{3}$, B. Kaiser ${ }^{4}$, N. Fiegl ${ }^{5}$, A. Artmann ${ }^{6}$, K. Hawlisch ${ }^{5}$, S. Newrkla ${ }^{7}$, M. Gessner ${ }^{8}$, R. Bernt ${ }^{9}$, J. Schuler ${ }^{10}$, G. Friedrich ${ }^{11}$, T. Trieb ${ }^{12}$, F. Wolf ${ }^{13}$, G. Reiter ${ }^{14}$, E. Sorantin ${ }^{15}$, C. Loewe ${ }^{13}$, A. Gamillscheg ${ }^{16}$

Die Institutsangaben sind am Ende des Beitrags gelistet.
Key words

- cardiac

- MR-imaging

- heart

eingereicht 18.6.2012

akzeptiert $\quad 6.8 .2012$

Bibliografie

Dol http://dx.doi.org/

10.1055/s-0032-1330763

Online-Publikation: 2013

Fortschr Röntgenstr 2013; 185 :

209-218 @ Georg Thieme

Verlag KG Stuttgart · New York .

ISSN 1438-9029

Korrespondenzadresse

Klaus Hergan

Universitätsinstitut für

Radiologie, Universitätsklinikum Salzburg

Müllner Hauptstrasse 48

5020 Salzburg

Österreich

Tel.: ++43/662/44823959

Fax: ++ 43/6 62/44823964

k.hergan@salk.at

\section{Zusammenfassung \\ $\nabla$}

Obwohl in den letzten Jahren wissenschaftlich weitere Indikationen für die Magnetresonanztomografie (MRT) des Herzens erarbeitet wurden, hat sich die Akzeptanz dieser Untersuchungstechnik in Klinik und Praxis bei den Kardiologen und Radiologen nicht mit der gleichen Geschwindigkeit entwickelt wie die diagnostischen Möglichkeiten. Verantwortlich dafür sind unter anderem die notwendige kostspielige Ausstattung, die relativ lange Untersuchungszeit, das hohe Maß an technischem Wissen und nicht zuletzt die fehlende oder unzureichende Remuneration. Darüber hinaus sind ein Festhalten an traditionellen Abklärungsalgorithmen, ein limitierter Zugang zu dieser Technologie sowie Probleme in der fächerübergreifenden Kooperation zu beobachten. Die seit vielen Jahren bestehende interdisziplinäre Kooperation hat die Aufgabe, einen substanziellen Beitrag zur Überwindung der genannten Problemfelder zu leisten und die MRT des Herzens in das Abklärungsspektrum kardialer Erkrankungen als fixen Bestandteil zu integrieren. Eine kontinuierliche Verbesserung der Behandlung von Patienten mittels kosteneffizienter Diagnostik und Therapie hat hierbei höchste Priorität. Die MRT des Herzens spielt dabei eine bedeutende Rolle.

\section{Einleitung \\ $\nabla$}

Diese Publikation befasst sich mit der kontinuierlichen Weiterentwicklung der Magnetresonanztomografie (MRT) des Herzens. Die MRT wie die CT stellen heute bei bestimmten kardiologischen Fragestellungen die Untersuchungsmethode der ersten Wahl dar [1, 2] und tragen entscheidend zur Therapieplanung bei ( $\$$ Tab. 1 adaptiert nach [3, 4]. Zu den Vorteilen der MRT zählen unter anderen: fehlende Invasivität und Strahlenexposition, beliebige Schichtführung, gleichzeitige Beurteilung

\section{Abstract \\ $\nabla$}

During the last years the indications of Cardiac Magnetic Resonance Imaging (CMRI) have been continuously expanded. However, the acceptance of the method by cardiologists and radiologists does not correlate with respect to the diagnostic potential. Several factors, such as expensive equipment, relatively long examination times, high technical know how and lack of remuneration, limit the application of CMRI in everyday clinical practice. Furthermore, doctors tend to apply more conventional, well established diagnostic procedures, the access to the method is still limited and there exist difficulties in the interdisciplinary collaboration. The interdisciplinary Austrian approach to Cardiac Imaging is aimed to improve the aforementioned problems and to support the implementation of CMRI in the diagnostic tree of cardiac diseases thus enabling a cost efficient management of patients in cardiology. 


\begin{tabular}{|c|c|c|c|c|}
\hline \multicolumn{5}{|c|}{ Klasse 2: MR ist gleich wie andere bildgebende Verfahren; Alternative; Entscheidungshilfe } \\
\hline \multicolumn{5}{|c|}{ Klasse 3: MR primär nicht indiziert, da andere Verfahren besser } \\
\hline \multicolumn{5}{|c|}{ Klasse Inv (investigational): MR ist derzeit noch in Erprobung, zu wenig Daten vorhanden } \\
\hline КнК & Morphologie & Funktion & $\begin{array}{l}\text { LE } \\
\text { (Late Enhancement) }\end{array}$ & Perfusion \\
\hline Nachweis KHK & 3 & 2 & 1 & 1 \\
\hline chron. Infarkt & 3 & 2 & 2 & 2 \\
\hline Postintervention & 3 & 2 & 2 & 2 \\
\hline nach Bypass & 3 & 2 & 2 & 2 \\
\hline \multicolumn{5}{|l|}{ Kardiomyopathie } \\
\hline HCMP, DCMP & 1 & 1 & 1 & \\
\hline RCMP & 1 & 2 & 1 & \\
\hline ARVD & 1 & 1 & 1 & \\
\hline \multicolumn{5}{|l|}{ Entzündung } \\
\hline Myokarditis & 1 & 1 & 1 & \\
\hline Perikarditis & 1 & 1 & 1 & \\
\hline konstr. Perikarditis & 1 & 1 & 1 & \\
\hline Perikarderguss & 1 & 1 & 1 & \\
\hline \multicolumn{5}{|l|}{ Raumforderung } \\
\hline Herz & 1 & 1 & 1 & \\
\hline Thrombus & 1 & 2 & 1 & \\
\hline Perikard & 1 & 1 & 1 & \\
\hline Klappen & & & Fluss & \\
\hline Stenose & 3 & 2 & 3 & \\
\hline Insuffizienz & 3 & 2 & 1 & \\
\hline paravalv. Abszess & Inv & 3 & Inv & \\
\hline Endokarditis & Inv & 3 & 3 & \\
\hline \multicolumn{5}{|l|}{ Aorta } \\
\hline Dissektion & 2 & 2 & & \\
\hline Aneurysma & 2 & 2 & & \\
\hline Isthmusstenose & 2 & 2 & & \\
\hline
\end{tabular}

MR-Indikationen (adaptiert nach $[3,4]$ ).

Ausgewählte Indikationen zum Herz-MR. Die Nummern entsprechen den Indikationsklassen. In der Regel sollte vor einem Herz-MR eine Echokardiografie erfolgt sein und daraus die Indikation zur MR-Untersuchung des Herzens entstehen.

auch prognostische Informationen geliefert werden. Im folgenden Beitrag werden die relevanten Indikationsgebiete dargestellt, auf physikalische Grundlagen und Details von Untersuchungsprotokollen wird nicht zuletzt wegen der kontinuierlichen technischen Weiterentwicklung bewusst verzichtet.

\section{Diagnostik der koronaren Herzerkrankung (KHK)}

Bei Patienten mit KHK können mittels MRT folgende Aspekte diagnostisch abgedeckt werden:

1. Myokardperfusion in Ruhe und unter pharmakologischer Belastung

2. Globale und regionale Wandbewegungsanalyse

3. Vitalität zur Indikationsstellung betreffend Revaskularisation

1. Myokardperfusion in Ruhe und unter pharmakologischer Belastung

Alternativ zur etablierten Myokardszintigrafie kann die Herz-MRT mittels Stressperfusion das Vorhandensein von ischämischen Myokardabschnitten bei signifikanten Koronarstenosen dokumentieren. Der Vorteil gegenüber der Szintigrafie liegt nicht nur in der räumlichen Auflösung und der Vermeidung der Strahlenbelastung, sondern auch in der kürzeren Untersuchungsdauer.

Grundlage der Ischämiediagnostik mittels MRT ist die Darstellung der Passage eines paramagnetischen Kontrastmittelbolus durch das Myokard unter gleichzeitiger Anwendung eines pharmakologischen Stressors (z. B. Adenosin oder Dipyridamol).
In der im Jahre 2012 veröffentlichen Metaanalyse [5] von 28 MRT-Studien (2970 Patienten), 10 Kontrastechostudien (795 Patienten) und 13 SPECT-Studien (1323 Patienten) war die Sensitivität und Spezifität der MRT verglichen mit der Koronarangiografie mit $91 \%$ und $80 \%$ signifikant besser als die der Echo- $(87 \%$ und $72 \%$ ) und SPECT-Studien (83\% und 77\%). Die Ergebnisse bestätigen die diagnostische Genauigkeit einer früheren Metaanalyse [6]. Adenosin als pharmakologisches Stress-Agens erzielt dabei eine gering höhere Sensitivität und Spezifität als Dipyridamol ( $90 \%$ u. $81 \%$ versus $86 \%$ u. $77 \%$ ). Interessant ist, dass die visuelle Beurteilung keine Nachteile gegenüber einer semiquantitativen Datenanalyse bedeutet [5], die Sensitivität sogar höher ist (visuell: Sensitivität $91 \%$, Spezifität 79\% versus semiquantitativ: 82 bzw. 84\%). Während sich in der MR-IMPACT-II-Multicenterstudie [7] die Stressperfusion mittels MRT der Myokardszintigrafie hinsichtlich der Sensitivität auf Kosten der Spezifität überlegen zeigte, war in der CE-MARC-Studie [8] die Sensitivität der Stressperfusion mittels MRT signifikant besser als Myokardszintigrafie, während die Spezifität identisch war. Die diagnostische Genauigkeit der Adenosin-Stress-MRT wird in einer weiteren Arbeit [9] bestätigt, in der die MRT mit der Druckdrahtmessung (fractional flow reserve- FFR) während einer Koronarangiografie verglichen wird und eine Sensitivität und Spezifität von 91 bzw. 94\% aufweist.

Bei Patienten mit koronarer Dreigefäßerkrankung ist die MRTStress-Perfusion mittels Adenosin aufgrund der höheren räumlichen Auflösung der nuklearmedizinischen Technik überlegen, wie eine Studie mit angiografisch verifizierter Dreigefäßerkran- 
kung zeigt [10]. Diese Arbeit belegt, dass eine signifikant höhere Anzahl an Myokardischämie-Arealen mittels MRT detektiert werden kann als mittels Myokardszintigrafie (84,6 versus 55,1\%, $\mathrm{p}<0,001$ in 3 Gefäßgebieten).

Standardisiert sollte neben der Stress-Perfusion eine Ruhe-Perfusion und eine „Late Enhancement“-Bildgebung durchgeführt werden, um wahre Perfusionsdefekte von möglichen Artefakten zu unterscheiden, was die Spezifität erhöht. Zusätzlich kann im Rahmen eines akuten Koronarsyndroms wie auch im Late Enhancement eine mikrovaskuläre Obstruktion in der Ruhe-Perfusionsstudie als subendokardiale dunkle Zone im pathologisch kontrastmittelverstärkten betroffenen Myokardareal dargestellt werden, was ein prognostischer Marker für zukünftige kardiale Ereignisse ist [11].

\section{Funktionsanalyse}

Die MRT ist der Goldstandard für die Ventrikelfunktionsanalyse. Die Berechnung der enddiastolischen und endsystolischen Volumina des rechten und linken Ventrikels erfolgt primär an aufeinanderfolgenden Kurzachsenschnitten, welche die Ventrikel komplett erfassen, wobei jede einzelne Schicht manuell oder semiautomatisch planimetriert wird. Zu beachten ist, dass die Papillarmuskeln dem Ventrikelvolumen hinzugerechnet werden. Durch zusätzliche epikardiale Konturierung ist für den linken Ventrikel eine Bestimmung der Muskelmasse zuverlässig möglich. Hervorzuheben ist die geringe Interobservervariabilität der Methode [12]. Globale und regionale Wandbewegungsstörungen können zuverlässig beurteilt und letztere dem etablierten 17Segmente-Modell zugeordnet werden [13]. Die fokalen Wandbewegungsstörungen werden als Hypokinesie, Akinesie und Dyskinesie klassifiziert. Bei der Beurteilung subtiler dyssynchroner Wandbewegungsstörungen ist die Sonografie bei entsprechender Schallqualität der MRT überlegen.

Die MR-Untersuchung des Herzens liefert ergänzend morphologische und funktionelle Informationen bei Komplikationen der KHK wie zum Beispiel dem ischämischen VSD, Ventrikelaneurysma und intrakardialen Thromben.

\section{Vitalitätsdiagnostik}

Durch die MR-tomografische Abbildung des Myokards mindestens 5 min nach Kontrastmittelgabe mithilfe einer Inversion-RecoverySequenz (Late Enhancement - LE) kann fibrotisches Gewebe im Myokard dargestellt werden. Dem Ausmaß von postischämischen Narben kommt als prädiktiver Faktor für das Outcome nach Revaskularisation große Bedeutung zu. Mit zunehmender Transmuralität einer Infarktnarbe wird eine funktionelle Erholung nach Revaskularisation unwahrscheinlicher $[14,15]$.

Für die Vorhersage der Funktionsverbesserung nach Revaskularisationstherapie kann ergänzend zur Narbendarstellung mittels LE auch eine Stress-MRT unter Gabe von Low-dose-Dobutamin hilfreich sein. Dies trifft insbesondere für Narben mit einem transmuralen Ausmaß von kleiner $75 \%$ zu $[16,17]$.

Eine zusätzliche Bedeutung hat die MRT des Herzens bei Patienten mit Herzinsuffizienz vor einer kardialen Resynchronisationstherapie (CRT-Schrittmachersystem) zur Verbesserung der linksventrikulären Auswurffraktion. Die Erfassung von Ausmaß und Lokalisation myokardialer Narben mittels LE ist für die Vorhersage des zu erwartenden Therapieerfolgs und die optimale Platzierung der linksventrikulären Schrittmachersonde bedeutend [18, 19].

\section{Nicht ischämische Erkrankungen des Myokards}

\section{$\nabla$}

\section{Kardiomyopathien}

Kardiomyopathien sind eine heterogene Gruppe von Krankheiten des Herzmuskels, die mit mechanischen, strukturellen und/oder elektrischen Funktionsstörungen bei fehlender koronarer Herzerkrankung, Hypertonie, Herzklappenerkrankung und angeborenen Herzfehlern einhergehen und eine unangemessene Hypertrophie des Myokards oder eine Dilatation der Herzkammern verursachen. Ihre Ursachen sind vielfältig und häufig genetisch bedingt. Kardiomyopathien begrenzen sich entweder auf das Herz oder sind Teil einer allgemeinen Systemerkrankung [20]. Die MR-Untersuchung des Herzens bietet bei exzellentem Blut- und Weichteilkontrast eine Fülle von diagnostischen und prognostischen Informationen $[21,22]$.

Bei Problemen in der echokardiografischen Bildgebung im Rahmen der Basisdiagnostik der Herzinsuffizienz sollte die MRT frühzeitig eingesetzt werden. Neben dem Nachweis und der Typisierung von Myokardschäden in der LE-Sequenz können mittels MRT auch inter- und intraventrikulären Leitungsstörungen in dynamischen Cinesequenzen dargestellt und eine Vorhersage betreffend Ansprechen auf eine kardiale Resynchronisationstherapie (CRT) getroffen werden. So konnte gezeigt werden, dass der Erfolg einer CRT und die Überlebenswahrscheinlichkeit der Patienten schlechter sind, je höher die Narbenbelastung des Myokards ist. Eine Positionierung der linksventrikulären Sonde im Narbengewebe erzielt keinen verbesserten hämodynamischen Effekt [23, 24].

Die MRT kann weiterhin vor einer diagnostischen Myokardbiopsie hilfreich bei der Wahl der Biopsiestelle sein [25]. Man unterscheidet morphologisch dilatative, hypertrophe, restriktive, arrhythmogene rechtsventrikuläre und unklassifizierte Kardiomyopathien. Eine weitere Einteilung erfolgt in familiär gehäufte (genetisch bedingte) und nicht familiäre (nicht genetisch bedingte) Formen [26].

Bei Kardiomyopathien sollten folgende Punkte im Befund besonders berücksichtigt werden:

1. Beurteilung der globalen und regionalen Funktion beider Ventrikel

2. Morphologische Beschreibung und Gewebscharakterisierung des Myokards

3. Beurteilung begleitender Pathologien (Perikarderguss, Fettinfiltrationen etc.)

\section{Differenzialdiagnose dilatative Kardiomyopathie (CMP)} versus ischämische CMP

Bei Patienten mit reduzierter linksventrikulärer Pumpfunktion kommt der Differenzialdiagnose zwischen dilatativer Kardiomyopathie (CMP) und ischämischer CMP eine fundamentale Bedeutung im Hinblick auf das therapeutische Management zu [27]. Mittels Echokardiografie ist diese Unterscheidung in der Regel nicht möglich, da das Kriterium der regionalen Wandbewegungsstörung nicht auf ischämische Formen der CMP beschränkt ist. Durch Einsatz der kontrastmittelverstärkten MRT können umschriebene intramyokardiale Fibroseherde oder Striae dargestellt werden. Bei der dilatativen CMP treten diese gehäuft intramyokardial im Ventrikelseptum oder subepikardial auf, bei der ischämischen CMP sind diese typischerweise subendokardial bis transmural lokalisiert. Eine eindeutige Differenzierung hinsichtlich einer ischämischen oder nicht ischämischen (z. B. postinfektiös oder toxisch) Genese ist aber auch durch das Muster der Kontrastmittelanreicherung nicht immer möglich. 


\section{Charakterisierung der hypertrophen Kardiomyopathie (HCMP)}

Die hypertrophe CMP ist eine genetisch bedingte Erkrankung der Actin- und Myosinfilamente und kann verschiedene Ausprägungsformen haben [28]. Die MRT erlaubt durch ihre dreidimensionale Erfassung beider Ventrikel in jeder beliebigen Schichtführung eine exakte Lokalisation der hypertrophierten Myokardareale und ist vor allem bei der Diagnose der apikalen HCMP der Echokardiografie deutlich überlegen. Ein variabel auftretendes LE-Muster ist fleckförmig, meist intramural in hypertrophierten Arealen oder im Ventrikelseptum und an der RV-Insertionsstelle im Septum [28]. In einigen Studien konnte auch ein Zusammenhang zwischen dem Nachweis einer pathologischen intramyokardialen Kontrastmittelanfärbung und der Prognose des Patienten gezeigt werden. Zusätzlich kann bei der hypertroph obstruktiven CMP eine Flussbeschleunigung in der Obstruktion visualisiert werden [29].

\section{Diagnose der Non-compaction-Kardiomyopathie}

Dies ist eine seltene Form einer CMP mit embryologisch bedingter fehlender Apposition der einzelnen Myokardschichten und konsekutiver Ausbildung tiefer Fissuren und Lakunen an der luminalen Seite des Myokards [30]. Besonders bei der apikalen Form können diese tiefen Lakunen sonografisch eine Pseudohypertrophie vortäuschen. Mittels MRT gelingt eine exakte anatomische Darstellung inklusive möglicher Thromben sowie die Differenzierung zwischen einer apikalen HCM, leichter apikaler Hypertrabekulierung ( $\leq 3$ Trabekel) und Non-compaction-CMP. Das Verhältnis Non-compacted/compacted-Myokard $\geq 2,3: 1$ diastolisch im 4-Kammerblick besitzt für diese Erkrankung eine hohe Sensitivität (85\%) und Spezifität (99\%) [31]. Die MRT des Herzens ist in der Lage, Fibrosebezirke darzustellen [32].

\section{Diagnose der stressassoziierten Kardiomyopathie (Tako-Tsubo)}

Bei dieser unklassifizierten Herzmuskelerkrankung, die häufig nach emotionalen Stresssituationen und überwiegend bei Frauen auftritt, und sowohl im Beschwerdebild hinsichtlich der EKGVeränderungen als auch der Laborwerte wie ein akuter Herzinfarkt imponiert, zeigt sich typischerweise eine aneurysmatische Auswölbung und Akinesie des Apex und in variablen Teilen der Vorderwand. In der Koronarangiografie finden sich keine signifikanten Koronarstenosen oder -verschlüsse. Mithilfe T2-gewichteter Sequenzen kann ein intramyokardiales Ödem dargestellt werden, ein ischämietypisches LE wird in der Regel nicht gefunden [33]. Neben der genauen Beschreibung der betroffenen linksventrikulären und selten auch rechtsventrikulären Myokardsegmente kann mit der MRT im Gegensatz zur Echokardiografie das Vorhandensein von thrombotischen Massen frühzeitig erfasst werden [34].

\section{Diagnose der restriktiven Kardiomyopathie}

Im Rahmen seltener primärer/idiopathischer sowie sekundärer restriktiver Kardiomyopathien kommt es zu einer Versteifung des Myokards mit konsekutiver Füllungsbehinderung. Die häufigsten Formen der restriktiven CMP sind die infiltrativen Kardiomyopathien (Amyloidose), entzündlich-granulomatösen Kardiomyopathien (Sarkoidose), Speichererkrankungen (Hämochromatose, Morbus Fabry, Glykogenspeichererkrankungen), das Hypereosinophilie-Syndrom und die Endomyokardfibrose [35]. Auch Anthrazykline und andere chemotherapeutische Substanzen sowie die Strahlentherapie können den Herzmuskel schädigen. Bei den meisten sekundären Formen der restriktiven CMP finden sich in der MRT des Herzens unterschiedliche LE-Muster, die in der Differenzierung hilfreich sein können. So zeigt beispielsweise die kardiale Amyloidose ein inhomogenes und zeitlich variables LE-Muster, das damit pathognomonisch wird [36].

Von therapeutischer Konsequenz ist die Abgrenzung der restriktiven CMP von der Perikarditis constrictiva. Die MRT ist dabei durch den Nachweis eines intramyokardialen LE, die Abgrenzung des Perikards und der Beurteilung der Vorhofgröße hilfreich, sollte aber durch eine funktionelle Echo-Doppler-Untersuchung ergänzt werden [37].

\section{Myokarditis \\ $\nabla$}

Die klinische Diagnose der Myokarditis ist schwierig, die Symptome der Patienten sind häufig uncharakteristisch. Die Konstellation der Laborbefunde (einschließlich Virusserologie), des EKG wie auch der Echokardiografie sind unspezifisch und daher auch in der Diagnostik nicht sehr hilfreich. Die MR-Untersuchung des Herzens hat die Myokarditis-Diagnostik maßgeblich erweitert [38]. Gekennzeichnet ist die Myokarditis durch ein Ödem des Myokards in T2-gewichteten Sequenzen und durch kontrastanreichernde Infiltrate in den LE-Sequenzen $[39,40]$. Meist ist die laterale oder ventrale Wand des linken Ventrikels betroffen [41]. Die Infiltrate finden sich unabhängig von den vaskulären Territorien und liegen eher intramural und subepikardial, viel seltener subendokardial $[42,43]$. Sind Veränderungen dieser Art im Septum sichtbar, sollte man auch an eine Sarkoidose denken.

Die Myokardbiopsie spielt einerseits bei der akut fulminanten Myokarditis zum Nachweis einer behandelbaren Ursache (z.B. giant cell oder eosinophile Form), andererseits beim Nachweis einer chronischen Myokarditis eine Rolle, die sich in der MRT des Herzens allenfalls durch die Messung der Signalintensitätsänderung in der T2-gewichteten bzw. T1-gewichteten Spinecho-Sequenz vor und nach Kontrastmittelgabe darstellen lässt [44]. Die Bestimmung des Quotienten der Signalintensitäten aus Myokard und Skelettmuskel haben sich aufgrund der notwendigen Erfassung standardisierter gerätespezifischer T1- und T2-Werte in der Routine nicht bewährt. Die ursprüngliche Hoffnung, die Effektivität einer Biopsie durch eine präinterventionelle MRT zu verbessern, hat sich in einer rezenten Studie nicht bestätigt. Im Wesentlichen wird der Erfolg der Biopsie durch die Zahl der biventrikulären Biopsate erhöht [45]. Die MRT des Herzens hat zudem einen prognostischen Stellenwert in der Verlaufskontrolle der Myokarditis [46].

\section{Rechtsventrikuläre Myokarderkrankungen $\nabla$}

Die arrhythmogene rechtsventrikuläre Kardiomyopathie/Dysplasie (ARVC/D) ist eine seltene genetische Erkrankung, die mit einem progredienten Ersatz von Myozyten durch Fett- und Bindegewebe einhergeht.

Seit Einführung der MRT in die kardiale Bildgebung wurde die Methode primär zum Nachweis von pathologischem Fettgewebe innerhalb des Myokards verwendet [47]. Obwohl es dazu hervorragende pathologisch-anatomische Korrelationsstudien gibt, hat sich im klinischen Alltag gezeigt, dass bedingt durch inkonstante Bildqualität auf Spinechosequenzen und Fehlinterpretation von Artefakten die Sensitivität zur Erkennung von intramyokardialem Fettgewebe zwischen $22 \%$ und $67 \%$ liegt $[48,49]$ und dass eine erhebliche Interobservervariabilität vorliegt [50]. Des Weiteren gibt 
es verschiedene histologische Subtypen mit unterschiedlichem Anteil an Binde- und Fettgewebe. Aus diesen Gründen wurde in den rezenten Guidelines der SCMRI der Fettnachweis als Hauptkriterium für das Vorliegen einer ARVD gestrichen, dafür ist als weiteres Kriterium der Nachweis von Bindegewebe mittels später Kontrastmittelanreicherung unter Verwendung von Gadolinium (LE) eingeführt worden [51].

Auch andere Kriterien der ARVD wie umschriebene oder generalisierte Wandverdünnungen, dyskinetische Segmente (Aneurysmen) oder Erhöhung der rechtsventrikulären Füllungsvolumina mit reduzierter Auswurffraktion können mittels MRT exakt diagnostiziert werden. Entscheidend ist bei der regionalen Wandbewegungsanalyse, dass „Normvarianten“ der RV-Funktion berücksichtigt werden, um eine Überbefundung zu vermeiden. Dazu gehört ein „physiologisches“ endsystolisches Bulging eines dünnen, aber normokinetischen Wandabschnitts (typischerweise im Bereich des Moderatorbands) oder eine als „dyskinetisch“ imponierende Diaphragmalwand in der kurzen Achse bedingt durch die Bewegung der Ventilebene relativ zur Schnittebene (through-plane motion). Ein Ausschluss einer ARVD/C kann mittels MRT alleine nicht erfolgen. Zur endgültigen Diagnose sind die internationalen Task-Force-Kriterien anzuwenden [51].

Eine eigene Entität stellt die benigne rechtsventrikuläre Ausflusstrakttachykardie dar, bei der es meist belastungsinduziert zu anhaltenden steiltypischen linksschenkelblockartigen ventrikulären Tachykardien kommt, die in der Regel nicht zu Synkopen oder zum plötzlichen Herztod führen. Bei diesem seltenen Krankheitsbild konnten mittels MRT-Veränderungen im Bereich des rechten Ventrikels gefunden werden, die an milde Formen der ARVD erinnern [52].

\section{Erkrankungen des Perikards \\ $\nabla$}

\section{Charakterisierung von Perikardergüssen}

Mittels MRT kann durch Anwendung unterschiedlicher Pulssequenzen eine Differenzierung zwischen serösen oder hämorrhagischen Ergüssen anhand der unterschiedlichen Signalintensitäten getroffen werden. Darüber hinaus ist eine Abschätzung des Ergussvolumens möglich. Speziell bei Patienten mit lokalisierten Ergüssen oder begleitenden Pathologien (abnorme Fettinfiltration oder perikardiale Tumore) ist die MRT der Echokardiografie deutlich überlegen, die aber die Diagnostik der ersten Wahl darstellt [53].

\section{Perikarditis}

Die MRT hat eine hohe Sensitivität bei der Diagnosestellung einer Perikarditis. Dabei helfen morphologische Kriterien wie Perikarddicke ( $>3 \mathrm{~mm}$ ), Perikarderguss (ev. lokalisiert) sowie eine Kontrastmittelanreicherung. Eine mögliche entzündliche Mitbeteiligung des Myokards lässt sich dadurch ebenfalls erfassen.

\section{Konstriktive Perikarditis}

Die konstriktive Perikarditis ist Folge eines chronischen Entzündungsprozesses, einer Herzoperation oder einer Radiatio und kann verkalkt oder nicht verkalkt in Erscheinung treten. Mittels MRT gelingt eine exakte Darstellung des verdickten Perikards $(>3 \mathrm{~mm})$ sowie eine Quantifizierung der kleinen Ventrikelvolumina und der erhöhten Vorhofvolumina [54]. Typisch ist eine Veränderung der Ventrikelgeometrie mit gestrecktem Ventrikelseptum und Impression der freien rechtsventrikulären Vorderwand. Der Schweregrad der konsekutiven Einflussstauung kann durch Diameterbestimmung der beiden Hohlvenen abgeschätzt werden. Eine normale Perikarddicke $(1-3 \mathrm{~mm})$ schließt eine konstriktive Perikarditis aus. Mittels MRT-Tagging-Technik kann die relative Bewegungsdifferenz zwischen dem starren Perikard und dem hyperdynamen Myokard veranschaulicht werden. Ähnlich wie in der Echokardiografie kann mittels Real-Time-MRT in In- und Exspiration der typische Shift des interventrikulären Septums dargestellt werden.

Bei der kalzifizierten Perikarditis ist aufgrund des fehlenden MRSignals von Kalk eine ergänzende Computertomografie sinnvoll, sofern nicht bereits die native Röntgenuntersuchung eine Perikardverkalkung nachgewiesen hat. Differenzialdiagnostisch können mittels MRT perikardiale Adhäsionen unterschieden werden, die je nach Ausprägungsgrad zu einer Behinderung der RV-Funktion führen können [53].

\section{Benigne Zysten}

Perikardzysten treten typischerweise im Bereich beider HerzZwerchfellwinkel auf und zeigen einen Kontakt zu Perikard und/ oder Diaphragma. Sie sind glatt begrenzt und weisen ein charakteristisches Signalverhalten auf (bei niedrigem Eiweißgehalt hypointens auf T1- und hyperintens auf T2-gewichteten Spinechosequenzen, starke Signalzunahme auf Inversionspulssequenzen) [55].

\section{Herztumore \\ $\nabla$}

Die häufigsten intrakardialen Raumforderungen sind Thromben. Sie sind besonders im Apexbereich mittels Echokardiografie gelegentlich nicht darstellbar. Diese Thromben können mithilfe der MRT zuverlässig dargestellt werden. Das Signalverhalten kann einen Hinweis auf das Alter des Thrombus geben.

Primäre Herztumore sind mit 0,1\% aller Tumore sehr selten [56]. Der häufigste benigne Tumor ist das Myxom, welches meist im Bereich der Vorhöfe lokalisiert ist [57]. Charakteristischerweise sitzt das Vorhofmyxom gestielt am interatrialen Septum und prolabiert abhängig von der Größe und Mobilität diastolisch in oder durch die AV-Klappenebene. Morphologisch ist das Myxom glatt begrenzt mit inhomogenem Signalverhalten (regressive, zystische Areale) und zeigt nach Kontrastmittelgabe eine variable Signalzunahme.

Primär maligne Herztumore sind vor allem diverse Sarkome. Diese weisen ein infiltratives Wachstum mit begleitendem, hämorrhagischem Erguss auf. Je nach Vaskularisationsgrad kommt es nach KM-Gabe zu einer Anreicherung mit Signalanhebung. Der diagnostische Vorteil der MRT liegt vor allem in der gleichzeitigen Beurteilung der parakardialen Strukturen. Dies ist besonders hilfreich bei der Zuordnung von Metastasen.

Als tumorös imponieren gelegentlich die fokale HCMP, Sarkoidose, Amyloidose sowie anatomische Varianten (Crista terminalis etc.).

Der hohe Stellenwert der MRT des Herzens liegt in der Differenzierung, Lokalisierung und ggf. Operationsplanung echokardiografisch detektierter raumfordernder Läsionen.

\section{Klappenerkrankungen \\ $\nabla$}

Während bei Klappenstenosen mittels Doppler-Sonografie und Herzkatheter Druckgradienten und Klappenöffnungsflächen exakt bestimmt werden können, stellt die Quantifizierung von Klappeninsuffizienzen nach wie vor eine diagnostische Heraus- 
forderung dar, da die meisten Methoden nur eine semiquantitative Beurteilung des Schweregrads erlauben. Die MRT hat auf diesem Gebiet das diagnostische Spektrum erweitert [58-60] und dient dazu, die Operationsindikation zu stellen oder zu festigen. Bei singulären Klappeninsuffizienzen kann aus dem Vergleich von links- und rechtsventrikulärem Schlagvolumen die Regurgitationsfraktion an der Herzklappe bestimmt werden. Exakter und vor allem, wenn mehrere Klappen insuffizient sind, kann durch Anwendung der Phasenkontrasttechnik in der Aorta ascendens und dem Pulmonalarterienhauptstamm die Differenz zwischen Vorwärtsschlagvolumen und Gesamtschlagvolumen aus der Volumetrie bestimmt werden [61 -65]. Die Aortenklappenstenose ist keine primäre Indikation zur MRT, sondern nur bei unklaren oder diskrepanten echokardiografischen Befunden kann eine planimetrische Bestimmung der Aortenöffnungsfläche hilfreich sein [66]. Die Pulmonalklappenerkrankungen und deren Folgen lassen sich mittels kardialer MRT sehr gut abklären bzw. die Interventions-/Operationsindikation besser abschätzen als mit anderen Methoden.

\section{Endokarditis \\ $\nabla$}

Die Echokardiografie (bevorzugt die transösophageale Untersuchung) ist gemeinsam mit klinischen und mikrobiologischen Befunden der Goldstandard in der Diagnose der Endokarditis. Die MRT spielt dabei eine untergeordnete Rolle. Eine myxomatös degenerierte Herzklappe kann in der MRT wie eine Endokarditis imponieren. Paravalvuläre Abszesse lassen sich mit der MRT erfassen, sind jedoch mittels CT des Herzens besser zu lokalisieren.

\section{Kongenitale Vitien im Kindesalter $\nabla$}

Angeborene Herzfehler finden sich in einer Inzidenz von knapp $<1 \%$ aller Neugeborenen [67]. Dank der enormen medizinischen Fortschritte und der damit gestiegenen Lebenserwartung dieser Patienten hat sich die Relation zwischen Kindern und Erwachsenen mit angeborenen Herzfehlern verschoben [67, 68]. So sind mittlerweile $80 \%$ der Patienten mit einer Fallot'schen Tetralogie älter als 20 Jahre. Die Echokardiografie ist mit zunehmendem Alter der Patienten limitiert. Insbesondere bei Funktionsuntersuchungen des rechten Ventrikels hat sich die MRT des Herzens mittlerweile als Goldstandard etabliert [70].

Die MRT des Herzens ist der Echokardiografie bei folgenden Fragestellungen überlegen: rechtsventrikuläre Volumetrie und Auswurffraktion, Evaluierung des rechsventrikulären Ausflusstrakts und von pulmonal-arteriellen Conduits, Quantifizierung der Pulmonalklappeninsuffizienz durch Bestimmung der Regurgitationsfraktion der Pulmonalklappe, Erfassung von peripheren Pulmonalarterienstenosen und Aneurysmen, Anatomie der Abklärung von System- und Pulmonalvenenanomalien bzw. deren Varianten und Fehlmündungen, Darstellung von komplexen Anomalien des Aortenbogens bzw. arteriovenösen Malformationen und Kollateralen, bei intra- und extrakardialen Raumforderungen/Tumoren, Bestimmung der biventrikulären Muskelmassen, Detektion und Quantifizierung von Myokardfibrosen sowie bei Gewebecharakterisierung (Fett, Eisen etc.).

Bei vermuteten Koronaranomalien (z. B. im Rahmen von belastungsinduzierten Synkopen) sollte bei jungen Patienten aufgrund der fehlenden Strahlenexposition die MRT zur Anwendung kom- men (Akquisition eines 3D-Datensatzes). Dies gilt auch für die Nachsorge bei Patienten mit Kawasaki-Syndrom.

\section{Kongenitale Vitien im Erwachsenenalter $\nabla$}

Seit Einführung der Herz-MRT stellen die kongenitalen Vitien ein Hauptindikationsgebiet dar. Besonders im Erwachsenenalter eignet sich die Methode zur Verlaufskontrolle bzw. zur Kontrolle von postoperativen Befunden und ersetzt - zusammen mit der Echokardiografie - in der Regel die Herzkatheteruntersuchung [71]. Dreidimensionale Analysen mithilfe der MRT erlauben eine exzellente Darstellung der oft komplexen postoperativen Anatomie.

Da mittels Spinechotechnik Vorhofseptumdefekte (ASD) übersehen oder überdiagnostiziert werden können, sind funktionelle Aufnahmen mit Gradientenechosequenzen erforderlich.

Die Beurteilung der rechtsventrikulären Morphologie, Funktionsund Volumenbestimmung ist eine Domäne der MRT. Die flusssensitiven Sequenzen erlauben eine Darstellung von Shuntflüssen und palliativ angelegten Gefäßverbindungen [72]. Ebenso kann ein Ventrikelseptumdefekt (VSD) nach Lokalisation klassifiziert werden (membranös, muskulär, supraapikal) und assoziierte Fehlbildungen (z. B. bikuspide Aortenklappe) können diagnostiziert werden.

Die Bestimmung von Shuntgrößen im Rahmen von fehlmündenden Lungenvenen, ASD, VSD, offenem Ductus Botalli und Koronarfisteln gelingt unter Verwendung der quantitativen Flussmessung mittels Phasenkontrasttechnik in Aorta und Pulmonalarterie. Daraus kann das Verhältnis von pulmonalem zu systemischem Blutfluss und damit die Shuntfraktion direkt bestimmt werden [72]. Im Falle einer begleitenden Pulmonalstenose mit systolischen Turbulenzen im Pulmonalishauptstamm kann eine Bestimmung des Blutflusses selektiv im linken und rechten Pulmonalgefäß erfolgen. Im Fall einer isolierten Pulmonalinsuffizienz kann einerseits aus der volumetrischen Differenz von links- und rechtsventrikulärem Schlagvolumen oder durch direkte Flussbestimmung im Pulmonalishauptstamm eine Regurgitationsfraktion bestimmt werden. Dies ist bei Patienten nach Totalkorrektur eines Morbus Fallot von besonderer klinischer Bedeutung [73].

Abgangsanomalien der Koronargefäße können eine hohe klinische Relevanz haben und können mittels MR-Angiografie (Akquisition eines 3D-Datensatzes) dargestellt werden.

\section{Pathologien der Aorta}

$\nabla$

\section{Aneurysmen und Dissektionen}

Bei klinisch stabilen Patienten stellt die MRT der Aorta eine Alternative zur CT-Angiografie bei der Abklärung, aber auch bei der Therapiekontrolle von Patienten mit Aortenaneurysmen und Aortendissektionen dar. Durch Kombination von anatomischen Bildern (Spinecho-Sequenzen mit und ohne Fettsättigung) mit flusssensitiven Sequenzen und kontrastverstärkter 3D-MR-Angiografie können mittels MRT alle relevanten Befunde (Aortendurchmesser, Wandbeschaffenheit, Thromben sowie Blutfluss im wahren und falschen Lumen) $[74,75]$ erhoben werden. Auch eine Stentgraftplanung zur Behandlung eines Aortenaneurysmas ist mit hochauflösenden, kontrastmittelverstärkten 3D-Sequenzen mit multiplanaren Reformationen möglich. Bei der Abklärung von thorakalen Aortenpathologien, wie zum Beispiel bei der Abklärung eines Aneurysmas des Sinus Valsalvae, können as- 
soziierte Klappenvitien mittels Gradientenecho nicht nur dargestellt, sondern durch Verwendung flusssensitiver Sequenzen auch quantifiziert werden.

\section{Kongenitale Aortenerkrankungen}

Aufgrund der Möglichkeit, anatomische und funktionelle Informationen nicht-invasiv in einer Untersuchung zu erfassen, spielt die MRT in der Abklärung angeborener Erkrankungen des Aortenbogens, welche im Erwachsenenalter einer Echokardiografie nur eingeschränkt zugänglich sind, eine tragende Rolle. Durch Kombination von anatomischer und funktioneller Bildgebung mittels MRT kann eine Aortenisthmusstenose diagnostiziert und die hämodynamische Relevanz quantifiziert werden. Der Kollateralfluss kann durch Zunahme des aortalen Blutvolumens von proximal nach distal mittels quantitativer Flussmessung bestimmt werden [76]. Zur Verlaufskontrolle von Patienten nach operativer Sanierung einer Aortenisthmusstenose ist die MRT die Methode der ersten Wahl. Demgegenüber ist nach Ballondilatation und Stentimplantation wegen möglicher MRT-Artefakte die CT vorzuziehen.

\section{Tesla-MRT in der Herzbildgebung}

Viele Anwendungen der Herz-MRT weisen technisch bedingt relativ geringe Signal- und Kontrast-zu-Rausch-Verhältnisse (SNR und CNR) auf, sodass die Durchführung der Herzbildgebung bei $3 \mathrm{~T}$ anstelle der etablierteren 1,5 T sehr vielversprechend erscheint [77, 78].

Die zunehmende Magnetfeldstärke bringt aber auch Effekte wie einen stärkeren magnetohydrodynamischen Effekt im EKG, geänderte magnetische Relaxationszeiten, geänderten "Chemical Shift", vermehrte Inhomogenitäten des anregenden Hochfrequenzfelds, höhere spezifische Absorptionsraten (SAR) bei gleichen Sequenzparametern und schlussendlich größere Magnetfeldinhomogenitäten mit sich [77, 78]. Besonders drastisch ist diese Situation bei der Steady-State-Free-Precession-Sequenz (SSFP), für deren Anwendung bei $3 \mathrm{~T}$ verbesserte Shim-Prozeduren [79, 80] und/oder eine über Scouting zu bestimmende Optimierung der Resonanzfrequenz [81] verwendet werden müssen, um Banding-Artefakte zu vermeiden.

Die Datenlage bzgl. einer möglichen Verbesserung der diagnostischen Genauigkeit der kardialen MRT bei $3 \mathrm{~T}$ ist sehr limitiert. Während die Gewinnung funktioneller Parameter ähnliche Ergebnisse bei 1,5 und $3 \mathrm{~T}$ liefert $[82,84]$, scheinen sich Vorteile von $3 \mathrm{~T}$ vor allem bei kontrastmittelunterstützten Techniken (Angiografien, Vitalitätsdiagnostik und Perfusion) abzuzeichnen. So konnte beispielsweise bei $3 \mathrm{~T}$ im direkten Patienten- und Feldstärkevergleich eine erhöhte Sensitivität, Genauigkeit und Spezifizität der Adenosin-Stress-Perfusion zum Nachweis von hämodynamisch relevanten Koronarstenosen bei Verdacht auf koronare Herzkrankheit gefunden werden [85]. Dies wird jedoch in einer aktuellen Metaanalyse [86] drastisch relativiert.

Berücksichtigt man schlussendlich noch die Tatsache, dass kardiale Implantate, sofern sie vorab getestet wurden, typischerweise ähnliche Sicherheitseigenschaften bei 1,5 und $3 \mathrm{~T}$ aufweisen [87], so lässt sich zusammenfassend wohl ein großes Potenzial der 3T-Herzbildgebung erkennen. Es bleibt abzuwarten, ob die Vorteile von $3 \mathrm{~T}$ gegenüber 1,5 T in der Herzbildgebung auch so eindeutig und bedeutend wie etwa bei der Neuro- oder Muskuloskelettal-Radiologie sein werden.

\section{Tab. 2 Kontraindikationen für eine Herz-MRT.}

absolute Kontraindikationen
Defibrillatoren, Herzschrittmacher und stillgelegte Elektroden
(Ausnahme siehe Text)
Neurostimulatoren
implantierte Pumpen
ferromagnetische Fremdkörper an kritischen Organen wie Gehirn,
Rückenmark, Augen
Hautklammern nach Operation, Vollgips
Starr-Edwards-Herzklappe
Swan-Ganz-Katheter, Hirndrucksonde, Harnkatheter mit
Temperatursonde
HAS-Schulterprothese
Zahnprothetik mit Magnetfixierung
relative Kontraindikationen
Implantate jeder Art, wenn diese nicht als „sicher“ eingestuft sind (Pass,
Website derFirma des Implantats), www.mri-safety.com, www.mrcomp.
com [92].

\section{Sicherheitsaspekte und Kontraindikationen der MRT} $\nabla$

Generell gelten für eine MRT des Herzens dieselben Ausschlusskriterien und Kontraindikationen wie für jede andere MR-Untersuchung ( Tab.2). Die heute implantierten Klappen, Stents, Stentgrafts und anderen herzspezifischen Implantate wie Coils oder Occluder sind im Allgemeinen MR-tauglich bzw. nach neuer Nomenklatur (bedingt) MR-sicher, in der Regel sogar unmittelbar nach Implantation, und stellen keine Kontraindikation gegen eine MRT dar. Subkutan implantierte Cardio-Rekorder sind ebenfalls bedingt MR-sicher, die relevanten Daten sollten jedoch vorher ausgelesen werden und spezifische Anweisungen des Herstellers müssen befolgt werden.

Im Gegensatz dazu stellen Herzschrittmacher und vor allem ICDSysteme prinzipiell primäre Kontraindikationen dar. Es ist nicht nur eine passagere und ggf. dauerhafte Funktionsstörung möglich, sondern es kann auch zu Spontanentladungen und zu Sondenerwärmung kommen. Bei niedrigen Feldstärken bis 1,5 Tesla ist unter kardiologischer Assistenz eine allgemeine MR-Untersuchung bei liegendem Herzschrittmacher möglich [88-90], jedoch als Routineverfahren abzulehnen.

Mittlerweile werden spezielle Herzschrittmacher- und Defibrillatorsysteme ohne Verwendung ferromagnetischer Materialien angeboten, welche als bedingt MR-sicher eingestuft sind und bei Einhaltung von bestimmten Vorgaben (Sicherheitsmaßnahmen, Umprogrammierung, energieadaptierte MR-Sequenzen) keine Kontraindikation darstellen [91].

Kontrastmittelallergie und Niereninsuffizienz sind relative Kontraindikationen, die teilweise medikamentös gut beherrschbar sind. Hier sind die Leitlinien der European Society of Urologic Radiology (www.esur.org) in der aktuellen Version zu beachten.

\section{Patientenvorbereitung}

\section{$\nabla$}

Grundsätzlich sind die allgemeinen Richtlinien bezüglich Patientensicherheit und Kontrastmittelsicherheit in der MRT wie bei allen anderen MRT-Untersuchungen anzuwenden. Speziell sollten die Patienten auf die lange Untersuchungsdauer (ca. $50 \mathrm{~min}$ ) und Sequenzen in Atemanhaltetechnik vorbereitet werden. Etwaige sprachliche Barrieren sind zu beachten. Bei pharmakologischer Stressuntersuchung sollten zwei venöse Zugänge vorhanden sein und die Patienten über die speziellen Vorkehrungen (Adenosin: 
Koffeinkarenz; Dobutamin: keine Beta-Blocker) aufgeklärt werden. Bei Patienten mit Klaustrophobie helfen teilweise Spiegelkonstruktionen oder eine alternative Lagerungstechnik. Eventuell ist die Anwesenheit einer Begleitperson im Untersuchungsraum hilfreich. Kinder dürfen ab dem 14. Lebensjahr selbstständig der Untersuchung zustimmen oder diese ablehnen (Teilrechtsfähigkeit).

\section{Zuweisung}

$\nabla$

Da die MRT des Herzens ein breites diagnostisches Spektrum bietet, sind eine klare Zuweisungsdiagnose und eine gezielte bzw. klinisch relevante Fragestellung notwendig. Die Zuweisung kann durch alle Fachbereiche erfolgen, nach entsprechender Konsultation des die MRT durchführenden Arztes zur Überprüfung der sinnvollen Indikation. Vorhandene kardiologische Vorbefunde (insbesondere eine Echokardiografie) und aktuelle Laborbefunde, insbesondere Kreatininwerte und glomeruläre Filtrationsrate vor Kontrastmittelgabe, sind beizulegen. Etwaige Kontraindikationen sind im Vorfeld abzuklären.

\section{Zusammenfassung}

Die MR-Untersuchung des Herzens hat sich als ergänzendes diagnostisches Verfahren etabliert und dient bei bestimmten Fragestellungen als Entscheidungshilfe im oberen Bereich der kardiologischen Diagnoseleiter. Mittels MRT können bei fast allen kardialen Erkrankungen relevante Zusatzinformationen gewonnen werden. Bei der Diagnostik der Myokarditis stellt die MRT die Methode der Wahl aller bildgebenden Verfahren dar. Die MRT hat bei der Vitalitätsdiagnostik einen zentralen Stellenwert zur Entscheidung betreffend Revaskularisation. Darüber hinaus kann mittels MRT eine sinnvolle Ischämiediagnostik angeboten werden. Im Rahmen der Abklärung von Kardiomyopathien kann die MRT wesentliche Informationen zur Genese liefern.

Eine weitere Verbreitung der Methode kann durch eine Standardisierung der Untersuchungsprotokolle, durch interdisziplinär abgestimmte Leitlinien zum Einsatz der MRT bei Erkrankungen des Herzens und durch qualifizierte Weiterbildung junger Ärzte erreicht werden.

\section{Institute}

\footnotetext{
1 Universitätsinstitut für Radiologie, Universitätsklinikum Salzburg

2 Herz-Kreislauf-Zentrum Groß Gerungs

${ }^{3}$ Department für Kardiologie/Intensivmedizin, Landeskrankenhaus Graz-West

${ }^{4}$ DZU, Diagnosezentrum Urania, Wien

${ }^{5}$ Kardiologie, KH Hietzing Wien

${ }^{6}$ Radiologisches Institut, Wels

Radiologie, SMZ Ost Wien

8 2. Medizinische Abteilung, Kardiologie, Hanusch Krankenhaus Wien

9 Radiologie, Hanusch-Krankenhaus

10 2. Medizinische Abteilung/Kardiologie, Universitätsklinikum Salzburg, Paracelsus Medizinische Privatuniversität

11 Universitätsklinik für Innere Medizin 3, Kardiologie, Medizinische Universität Innsbruck

12 Universitätsklinik für Radiologie, Medizinische Universität Innsbruck

13 Universitätsklinik für Radiodiagnostik, Medizinische Universität Wien

14 Siemens AG, Healthcare Division, Graz, Austria

15 Universitätsklinik für Radiologie, klin. Abteilung für Kinderradiologie, Medizinische Universität Graz

16 Universitätsklinik für Kinder- und Jugendheilkunde, klinische Abteilung f. pädiatrische Kardiologie, Medizinische Universität Graz
}

\section{Danksagung \\ $\nabla$}

Die Autoren bedanken sich bei Herrn Prim. Univ.-Prof. Dr. Herbert Frank, Leiter der Abteilung für Innere Medizin am Landeskrankenhaus Tulln und Herrn Prim. Univ.-Prof. Dr. Gerhard Mostbeck, Leiter des Zentralröntgeninstitutes am Wilhelminenspital und Otto Wagner Spital Wien, für die fachlich unabhängige Revision des Manuskripts.

\section{Literatur}

1 Achenbach S, Barkhausen J, Beer $M$ et al. Konsensusempfehlungen der DRG/DGK/DGPK zum Einsatz der Herzbildgebung mit Computertomografie und Magnetresonanztomografie. Fortschr Röntgenstr 2012; 184: $345-368$

2 Hergan K, Globits S, Loewe C et al. Aktueller Stellenwert der MSCTA in der Koronargefassdiagnostik (2011): Klinischer Leitfaden der Osterreichischen Gesellschaften fur Kardiologie und Radiologie. Fortschr Röntgenstr 2011; 183: 964-971

3 Lotz J, Kivelitz D, Fischbach R et al. Empfehlungen für den Einsatz der Computertomografie und Magnetresonanztomografie in der Herzdiagnostik. Teil 2 - Magnetresonanztomografie. Fortschr Röntgenstr 2009; 181: 800-814

4 Pennell DJ, Sechtem UP, Higgins CB et al. Clinical indications for cardiovascular magnetic resonance (CMR): Consensus Panel report. Eur Heart J 2004; 25: 1940 - 1965

5 de Jong MC, Genders TSS, van Geuns RJ et al. Diagnostic performance of stress myocardial perfusion imaging for coronary artery disease: a systemic review and meta-analysis. Eur Radiol (electronically published) . DOI: $10.1007 / \mathrm{s} 00330-012-2434-1$

6 Nandalur KR, Dwamena BA, Choudhri AF et al. Diagnostic performance of stress cardiac magnetic resonance imaging in the detection of coronary artery disease. J Am Coll Cardiol 2007; 50: 1343 - 1353

7 Schwitter J, Wacker CM, Wilke N et al. MR-IMPACT II: Magnetic resonance imaging for myocardial perfusion assessment in coronary artery disease trial: perfusion-cardiac magnetic resonance vs. single-photon emission computed tomography for the detection of coronary artery disease: a comparative multicentre, multivendor trial. Eur Heart J 2012, (first published online March 4, 2012)

8 Greenwood JP, Maredia N, Younger JF et al. Cardiovascular magnetic resonance imaging and single-photon emission computed tomography for diagnosis of coronary heart disease (CE-MARK): a prospective trial. Lancet 2012; 379: 453-460

9 Watkins S, McGeoch R, Lyne J et al. Validation of Magnetic Resonance Myocardial Perfusion Imaging With Fractional Flow Reserve for the Detection of Significant Coronary Heart Disease. Circulation 2009; 120: $2207-2213$

10 Chung SY, Lee KY, Chun EJ et al. Comparison of stress perfusion MRI and SPECT for detection of myocardial ischemia in patients with angiographically proven threevessel coronary artery disease. Am J Roentgenol 2010; 195: 356-362

11 Klug G, Mayr A, Schenk S et al. Prognostic value at 5 years of microvascular obstruction after acute myocardial infarction assessed by cardiovascular magnetic resonance. J Cardiovasc Magn Reson 2012; 14: 46

12 Grothues F, Smith GC, Moon JC et al. Comparison of interstudy reproducibility of cardiovascular magnetic resonance with two-dimensional echocardiography in normal subjects and in patients with heart failure or left ventricular hypertrophy. Am J Cardiol 2002; 90: 29-34

13 Cerqueira MD, Weissman NJ, Dilsizian V et al. Standardized Myocardial Segmentation and Nomenclature for Tomographic Imaging of the Heart: A Statement for Healthcare Proffessionals From the Cardiac Imaging Committee of the Council on Clinical Cardiology of the American Heart Association. Circulation 2002; 105: 539-542

14 Allman KC, Shaw LJ, Hachamovitch $R$ et al. Myocardial viability testing and impact of revascularization on prognosis in patients with coronary artery disease and left ventricular dysfunction: a meta-analysis. J Am Coll Cardiol 2002; 39: 1151 - 1158

$15 \mathrm{Kim} \mathrm{RJ}, \mathrm{Wu}$ E, Rafael A et al. The use of contrast-enhanced magnetic resonance imaging to identify reversible myocardial dysfunction. $\mathrm{N}$ Engl J Med 2000; 343: 1445-1453

16 Wellnhofer E, Olariu A, Klein C et al. Magnetic resonance low-dose dobutamine test is superior to scar quantification for the prediction of functional recovery. Circulation 2004; 109: 2172-2174 
17 Kim RJ, Manning WJ. Viability assessment by delayed enhancement cardiovascular magnetic resonance imaging: will low-dose dobutamine dull the shine? Circulation 2004; 109: 2476-2479

18 White JA, Yee R, Yuan X et al. Delayed enhancement magnetic resonance imaging predicts response to cardiac resynchronization therapy in patients with intraventricular dyssynchrony. J Am Coll Cardiol 2006; 48: $1953-1960$

19 Chalil S, Foley P, Muyhaldeen S et al. Late gadolinium enhancementcardiovascular magnetic resonance as a predictor of response to cardiac resynchronization therapy in patients with ischaemic cardiomyopathy. Europace 2007; 9: 1031 - 1037

20 Maron BJ, Towbin JA, Thiene G et al. Contemporary definitions and classification of the cardiomyopathies. A scientific statement of the American Heart Association. Circulation 2006; 113: 1807-1816

21 Karamitsos TD, Francis JM, Myerson S. The role of cardiovascular magnetic resonance imaging in heart failure. J Am Coll Cardiol 2009; 54 : $1407-1424$

22 Nathan M, Chia YL, Croisille P et al. Assessment of Myocardial Fibrosis With Cardiovascular Magnetic Resonance. J Am Coll Cardiol 2011; 57: $891-903$

23 Leyva $F$. Cardiac resynchronization therapy guided by cardiovascular magnetic resonance. J Cardiovasc Magn Reson 2010; 12: 64-86

24 Leyva F, Foley PW, Chalil $S$ et al. Cardiac resynchronisation therapy guided by late gadolinium-enhancement cardiovascular magnetic resonance. J Cardiovasc Magn Reson 2011; 13: 29 - 38

25 Pankuweit S, Richter A, Ruppert V et al. Classification of cardiomyopathies and indication for endomyocardial biopsy revisited. Herz 2009; 34: $55-62$

26 Elliott P, Andersson B, Arbustini E et al. Classification of the cardiomyopathies: a position statement from the european society of cardiology working group on myocardial and pericardial diseases. Eur Heart J 2008; 29: 270-276

27 McCrohon JA, Moon JC, Prasad SK et al. Differentiation of heart failure related to dilated cardiomyopathy and coronary artery disease using gadolinium enhanced cardiovascular magnetic resonance. Circulation 2003; 108: $54-59$

28 Gersh BJ, Maron BJ, Bonow RO et al. 2011 ACCF/AHA guideline for the diagnosis and treatment of hypertrophic cardiomyopathy: executive summary: a report of the American College of Cardiology Foundation/American Heart Association Task Force on practice guidelines developed in collaboration with the American Association for Thoracic Surgery, American Society of Echocardiography, American Society of Nuclear Cardiology, Heart Failure Society of America, Heart Rhythm Society, Society for Cardiovascular Angiography and Interventions, and Society of Thoracic Surgeons. J Am Coll Cardiol 2011; 58: 2703 2738

29 White JA, Fine NM, Gula L et al. Utility of cardiovascular magnetic resonance in identifying substrate for malignant ventricular arrhythmias. Circ Cardiovasc Imaging 2012; 5: $12-20$

30 Jenni R, Oechslin E, Schneider J et al. Echocardiographic and pathoanatomical characteristics of isolated left ventricular noncompaction: a step towards classification as a distinct cardiomyopathy. Heart 2001; 86: $666-671$

31 Petersen SE, Selvanayagam JB, Wiesmann F. Left Ventricular Non-Compaction: Insights From Cardiovascular Magnetic Resonance Imaging. J Am Coll Cardiol 2005; 46: 101 - 105

32 Dursun M, Agayev A, Ertugrul T et al. MR imaging features of ventricular noncompaction: Emphasis on distribution and pattern of fibrosis. Eur J Radiol 2010; 74: 147-151

33 Fernández-Pérez GC, Anguilar-Arjona JA, Tardáguilla de la Fuente G et al. Takotsubo cardiomyopathy: Assessment with cardiac MRI. Am J Roentgenol 2010; 195: W139-W145

34 Sharkey SW, Windenburg DC, Lesser JR et al. Natural History and Expansive Clinical Profile of Stress (Tako-Tsubo) Cardiomyopathy. J Am Coll Cardiol 2010; 55: $333-341$

35 Masui T, Finck S, Higgins $C B$. Constrictive pericarditis and restrictive cardiomyopathy: evaluation with MR imaging. Radiology 1992; 182: $369-373$

36 Mahrholdt H, Wagner A, Judd RM et al. Delayed enhancement cardiovascular magnetic resonance assessment of non-ischaemic cardiomyopathies. Eur Heart J 2005; 26: 1461 - 1474

37 Cheng $H$, Zhao $S$, Jiang $S$ et al. The relative atrial volume ratio and late gadolinium enhancement provide additive information to differentiate constrictive pericarditis from restrictive cardiomyopathy. J Cardiovasc Magn Reson 2011; 13: 15-23
38 Breuckmann F, Maderwald S, Buhr C et al. Cardiac MRI: estimation of changes in normalized myocardial gadolinium accumulation over time after contrast injection in patients with acute myocarditis and healthy volunteers. Fortschr Röntgenstr 2011; 183: 933-938

39 Friedrich MG, Sechtem U, Schulz-Menger J et al. Cardiovascular Magnetic Resonance in Myocarditis: A JACC White Paper. J Am Coll Cardiol 2009; 53: 1475 - 1487

40 Frank H, Globits S. Magnetic Resonance Imaging evaluation of myocardial and pericardial disease. JMRI 1999; 10: 617-626

41 Laissy JP, Hyafil F, Juliard JM et al. Differentiating acute myocardial infarction from Myocarditis: Diagnostic value of early- and delayed-perfusion cardiac MR imaging. Radiology 2005; 237: 75-82

42 Goitein O, Matetzky S, Beinart R et al. Acute myocarditis: non-invasive evaluation with cardiac MRI and transthoracic echocardiography. Am J Roentgenol 2008; 192: $254-258$

43 Gahide $G$, Bertrand D, Roubille F et al. MR delayed enhancement imaging findings in suspected acute myocarditis. Eur Radiol 2010; 20: $65-72$

44 Gutberlet $M$, Spors B, Thoma $T$ et al. Suspected chronic myocarditis at cardiac CT: diagnostic accuracy and association with immunohistological detected inflammation and viral persistence. Radiology 2008; 246: 401 - 409

45 Yilmaz A, Kindermann I, Kindermann $M$ et al. Comparative evaluation of left and right ventricular endomyocardial biopsy: differences in complication rate and diagnostic performance. Circulation 2010; 122 : 900-909

46 Mahrhold H, Goedecke C, Wagner A et al. Cardiovascular magnetic resonance assessment of human myocarditis: a comparison to histology and molecular pathology. Circulation 2004; 109: 1250-1258

47 Keller D, Osswald S, Bremerich J. Arrhythmogenic rightventricular dysplasia: diagnostic and prognostic value of cardiac MRI in relation to arrhythmia free survival. Int J Card Imaging 2003; 19: 537-543

48 Kayser HW, van der Wall EE, Sivananthan MU et al. Diagnosis of arrhythmogenic right ventricular dysplasie: a review. Radiographics 2002; 22: 639-648

49 Marcus FI, Zareba W, Calkins $H$ et al. Arrhytmogenic right ventricular cardiomyopathy/dysplasia clinical presentation and diagnostic evaluation: results from the North American Multidisciplinary Study. Heart Rhythm 2009; 6: 984-992

50 Bluemke DA, Krupinski EA, Ovitt T et al. MRI Imaging of arrhythmogenic right ventricular cardiomyopathy: morphological findings and intraobserver reliability. Cardiology 2003; 99: 153-612

51 Marcus FI, McKenna WJ, Sherrill D et al. Diagnosis of arrhythmogenic right ventricular cardiomyopathy/dysplasia: proposed modification of the task force criteria. Circulation 2010; 121: 1533-1541

52 Globits $S$, Kreiner G, Frank $H$ et al. Significance of morphological abnormalities detected by MRI in patients undergoing successful ablation of right ventricular outflow tract tachycardia. Circulation 1997; 96: $2633-2640$

53 Wang ZJ, Reddy GP, Gotway MB et al. CT and MR imaging of pericardial disease. Radiographics 2003; 23: S167-S180

54 Masui T, Finck S, Higgins $C B$. Constrictive pericarditis and restrictive cardiomyopathy: evaluation with MR imaging. Radiology 1992; 182 : $369-373$

55 Sechtem $U$, Tscholakoff $D$, Higgins $C B$. MRI of the abnormal pericardium. Am J Roentgenol 1986; 147: 239-244

56 Lam KY, Dickens P, Chan AC. Tumors of the heart. A 20-year experience with a review of 12,485 consecutive autopsies. Arch Pathol Lab Med 1993; 117: $1027-1031$

57 Hoffmann $U$, Globits S, Schima $W$ et al. Usefulness of magnetic resonance imaging of cardiac and paracardiac masses. Am J Cardiol 2003; 92: $890-895$

58 Aurigemma $G$, Reichek $N$, Schiebler $M$ et al. Evaluation of aortic regurgitation by cardiac cine MRI: planar analysis and comparison to Doppler echocardiography. Cardiology 1991; 78: 340-347

59 Wagner S, Auffermann W, Buser Pet al. Diagnostic accuracy and estimation of the severity of valvular regurgitation from the signal void on cine magnetic resonance imaging. Am Heart J 1989; 118: 760 - 767

60 Glockner JF, Johnston DL, McGee KP. Evaluation of cardiac valvular disease with MR imaging: Qualitative and quantitative techniques. Radiographics 2003; 23: e9

61 Nishimura F. Oblique cine MRI for the evaluation of aortic regurgitation: comparison with cineangiography. Clin Cardiol 1992; 15: $73-78$

62 Globits S, Frank F, Mayr H et al. Quantitative assessment of aortic regurgitation by magnetic resonance imaging. Eur Heart J 1992 13: 78 -83 
63 Sondergaard L, Lindvig K, Hildebrandt $P$ et al. Quantification of aortic regurgitation by magnetic resonance velocity mapping. Am Heart J 1993; 125: $1081-1090$

64 Fujita N, Chazouilleres AF, Hartiala JJ et al. Quantification of mitral regurgitation by velocity-encoded cine nuclear magnetic resonance imaging. J Am Coll Cardiol 1994; 23: 951 -958

65 Morris MF, Maleszewski IJ, Suri RM et al. CT and MR imaging of the mitral valve: radiologic-pathologic correlation. Radiographics 2010; 30 : $603-620$

66 Didier D. Assessment of valve disease. Qualitative and quantitative. Magn Reson Imaging Clin N Am 2003; 11: 115 - 134

67 Health Canada. Congenital Anomalies in Canada - A Perinatal Health Report. Minister of Public Works and Government Services Canada; 2002, http://www.phac-aspc.gc.ca/publicat/cac-acc02/index-eng.php

68 Marelli AJ, Mackie AS, Ionescu-Ittu $R$ et al. Congenital heart disease in the general population: Changing prevalence and age distribution. Circulation 2007; 115: $163-172$

69 Freeman $L J$. Grown up congenital heart (GUCH) disease: a half century of change. Clinical medicine 2008; 8: 169-171

70 Kilner PJ, Geva T, Kaemmerer $\mathrm{H}$ et al. Recommendations for cardiovascular magnetic resonance in adults with congenital heart disease from the respective working groups of the European Society of Cardiology. European Heart J 2010; 3: 794-805

71 Kersting-Sommerhoff BA, Diethelm L, Stanger P et al. Evaluation of complex congenital ventricular anomalies with magnetic resonance imaging. Am Heart J 1990; 120: 133-142

72 Hundley WG, Li HF, Lange RA et al. Assessment of left-to-right intracardiac shunting by velocity-encoded, phase-difference magnetic resonance imaging. A comparison with oximetric and indicator dilution techniques. Circulation 1995; 91: 2955-2960

73 Rebergen SA, Chin JG, Ottenkamp J et al. Pulmonary regurgitation in the late postoperative follow-up of tetralogy of Fallot. Volumetric quantitation by nuclear magnetic resonance velocity mapping. Circulation 1993; 92: 1123-1132

74 Sommer T, Fehske W, Holzknecht $N$ et al. Aortic dissection: a comparative study of diagnosis with spiral CT, multiplanar transesophageal echocardiography, and MR imaging. Radiology 1996; 199: 347-352

75 Fayad ZA, Nahar T, Fallon JT et al. In vivo magnetic resonance evaluation of atherosclerotic plaques in the human thoracic aorta: a comparison with transesophageal echocardiography. Circulation 2000; 101: $2503-2509$

76 Steffens JC, Bourne MW, Sakuma $H$ et al. Quantification of collateral blood flow in coarctation of the aorta by velocity encoded cine magnetic resonance imaging. Circulation 1994; 90: 937-943

77 Wieben 0 , Francois C, Reeder SB. Cardiac MRI of ischemic heart disease at 3 T: potential and challenges. Eur J Radiol 2008; 65: 15-28

78 Oshinski JN, Delfino JG, Sharma P et al. Cardiovascular magnetic resonance at 3.0 T: current state of the art. J Cardiovasc Magn Reson 2010; 12: $55-68$

79 Schär M, Kozerke S, Fischer SE et al. Cardiac SSFP imaging at 3 Tesla. Magn Reson Med 2004; 51: 799-806
80 Greiser A, Weber O, Deshpande V et al. Improved cardiac shimming in a clinical setting by multi-frame fieldmap acquisition and automatic ROI extension. J Cardiovasc Magn Reson 2007; 9: 239-240

81 Wansapura J, Fleck R, Crotty E et al. Frequency scouting for cardiac imaging with SSFP at 3 Tesla. Pediatr Radiol 2006; 36: 1082-1085

82 Michaely HJ, Nael K, Schoenberg SO et al. Analysis of cardiac function comparison between 1.5 Tesla and 3.0 Tesla cardiac cine magnetic resonance imaging: preliminary experience. Invest Radiol 2006; 41: $133-140$

83 Hudsmith LE, Cheng AS, Tyler DJ et al. Assessment of left atrial volumes at 1.5 Tesla and 3 Tesla using FLASH and SSFP cine imaging. J Cardiovasc Magn Reson 2007; 9: 673-679

84 Maroules $C D$, McColl $R$, Khera A et al. Interstudy reproducibility of SSFP cine magnetic resonance: impact of magnetic field strength and parallel imaging. J Magn Reson Imaging 2008; 27: 1139-1145

85 Cheng AS, Pegg TJ, Karamitsos TD et al. Cardiovascular magnetic resonance perfusion imaging at 3-tesla for the detection of coronary artery disease: a comparison with 1. 5-Tesla. J Am Coll Cardiol 2007; 49: $2440-2449$

86 Hamon M, Fau G, Née G et al. Meta-analysis of the diagnostic performance of stress perfusion cardiovascular magnetic resonance for detection of coronary artery disease. J Cardiovasc Magn Reson 2010; 12: $29-39$

87 Levine GN, Gomes AS, Arai AE et al. American Heart Association Committee on Diagnostic and Interventional Cardiac Catheterization; American Heart Association Council on Clinical Cardiology; American Heart Association Council on Cardiovascular Radiology and Intervention. Safety of magnetic resonance imaging in patients with cardiovascular devices: an American Heart Association scientific statement from the Committee on Diagnostic and Interventional Cardiac Catheterization, Council on Clinical Cardiology, and the Council on Cardiovascular Radiology and Intervention: endorsed by the American College of Cardiology Foundation, the North American Society for Cardiac Imaging, and the Society for Cardiovascular Magnetic Resonance. Circulation 2007; 116: $2878-2891$

88 Strach K, Naehle CP, Mühlsteffen A et al. Low field magnetic resonance imaging: increased safety for pacemaker patients? Europace 2009; 12 : $952-960$

89 Naehle CP, KreuzJ, Strach K et al. Safety, feasibility, and diagnostic value of cardiac magnetic resonance imaging in patients with cardiac pacemakers and implantable cardioverters/defibillators at 1.5T. Am Heart J 2011; 161: $1096-1105$

90 Rouguin A, Schwitter J, Vahlhaus C et al. Magnetic resonance imaging in individuals with cardiovascular implantable electronic devices. Europace 2008; 10: $336-346$

91 Sutton R, Kanal E, Wilkoff BL et al. Safety of magnetic resonance imaging of patients with a new Medtronic EnRhythm MRISureScan pacing system: clinical study design. Trials 2008; 9: 68-76

92 Shellock FG. Reference Manual for Magnetic Resonance Safety, Implants, and Devices 2011. Los Angeles: Biomedical Research Publishing Group 